\title{
Upgrade of the Lithium beam diagnostic at JET
}

M. Brix, ${ }^{1, a)}$ D. Dodt, ${ }^{2}$ A. Korotkov, ${ }^{1}$ P. Morgan, ${ }^{1}$ D. Dunai, ${ }^{3}$ R Fischer, ${ }^{2}$ A. Meigs, ${ }^{1}$ I. S. Nedzelskiy, ${ }^{4}$ J. Schweinzer, ${ }^{2}$ J. Vince, ${ }^{1}$ S. Zoletnik, ${ }^{3}$ and JET-EFDA Contributors ${ }^{\mathrm{b})}$

(JET-EFDA Culham Science Centre, Abingdon, OX14 3DB, UK)

1) JET-EFDA, EURATOM/CCFE Fusion Association, Culham Science Centre, Abingdon, OX14 3DB, $U K$

${ }^{2)}$ Max Planck Institut für Plasmaphysik, Association EURATOM/MPI, Garching, Germany

3) KFKI RMKI, EURATOM Association,Budapest, Hungary

4) Association EURATOM/IST, Inst. de Plasma e Fusão Nuclear, Inst. Superior Técnico, Lisboa, Portugal

(Dated: 12 May 2010)

A $60 \mathrm{kV}$ neutral Li beam is injected into the edge plasma of JET to measure the electron density. The beam observation system has been improved by replacing a Czerny-Turner spectrometer with a high-resolution transmission-grating spectrometer and a fast back-illuminated frame-transfer camera. The larger throughput of the spectrometer, the increased sensitivity and the faster readout of the new camera allow inter-ELM measurements (frame rate of $100 \mathrm{~Hz}$ ). The calibration of the setup, as well as an improved spectral fitting technique in the presence of carbon background radiation, is discussed in detail. The density calculation is based on a statistical analysis method. Results are presented for different plasma scenarios.

\section{INTRODUCTION}

The Li beam diagnostic is an active spectroscopic technique for the measurement of electron density profiles in the edge plasma of tokamaks ${ }^{1}$. Fast Li beams with energies of 20 to $70 \mathrm{kV}$ can be created by extraction from thermionic emitters and collimated and accelerated to high beam velocities by a set of extraction grids. A Li beam diagnostic has been in operation at JET for more than 10 years $^{2}$, the beam emission of the Li beam atoms is detected by a spectrometer-based observation system. The upgrade of this observation system is the main subject of this paper. A transmission grating spectrometer with high throughput has replaced a Czerny-Turner spectrometer, a 15 year old Wright instruments CCD camera has been replaced by a-state-of-the-art Princeton Instrument PhotonMax camera with fast readout and high quantum efficiency. Both replacements have significantly enhanced the sensitivity of the observation system and hence allow for an improved signal-to-noise ratio and for a higher temporal resolution of the beam emission measurement.

The outline of the paper is as follows: first the experimental setup and the observation system at JET are described in some detail. A discussion of the spatial, spectral and intensity calibration is followed by an explanation of the spectral fitting technique. Finally, the resulting calibrated beam-emission data are used to derive profiles for electron density for different plasma scenarios.

\footnotetext{
a) mathias.brix@ccfe.ac.uk

b) See the Appendix of F. Romanelli et al., Proceedings of the 22nd IAEA Fusion Energy Conference 2008, Geneva, Switzerland
}

\section{THE LI BEAM DIAGNOSTIC AT JET}

The Li beam gun is located above the transformer limbs on top of the tokamak at a radius of $3.25 \mathrm{~m}$ and approximately $4.9 \mathrm{~m}$ above the plasma boundary. The JET Li ion gun is similar to the ASDEX design ${ }^{1,2}$; details on the JET beam diagnostics are described in these proceedings ${ }^{3}$.

At ASDEX Upgrade, DIIID and Textor, the Li beam is injected into the midplane of the tokamak. The plasma boundary at the midplane is located usually close to a poloidal limiter, hence the observation system can be designed for a fixed position. In contrast, the Li beam at JET probes the boundary on the top of the plasma. A variety of different plasma shapes is investigated at JET, the location of the seperatrix changes by more than $25 \mathrm{~cm}$ at the Lithium beam position, hence the observation system has to be adjusted to different plasma edge positions. The optical components are installed in a periscope tube, which is located at the same radial position but seperated by a toroidal distance of $\mathrm{d}=65 \mathrm{~cm}$. The plasma facing side of the periscope is equipped with a tiltable mirror to adjust the measurement area to the region of interest. In closed position, the mirror doubles as a protective shutter for the plasma facing window. The spatial calibration of the observation system is performed by measuring the Doppler shift of the Li beam emission, hence, a spectrometer/CCD camera detection system is required. Spatially fixed observation systems can use a combination of narrow interference filters and fast detectors like avalanche diodes or photo multipliers. Whereas the latter systems have a larger throughput and allow for a better signal-tonoise and/or faster temporal resolution, the spectrometer setup at JET not only measures the intensity of the beam emission but also provides the spatial calibration and a spectrally-resolved detection of the background radiation. 
